\title{
Solving an X-ray structure of a bio-artificial neurotransmitter receptor designed for the naked-eye recognition of dopamine
}

\author{
Thibaud Rossel ${ }^{a, 1,2}$, Bing Zhangb,3, Raphael Gobat ${ }^{\mathbf{b}}$ \\ a Gymnase français de Bienne, \\ Rue du débarcadère 8,2503 , Bienne, Switzerland \\ ${ }^{b}$ Biozentrum, University of Basel, \\ Klingelbergstrasse 50/70, Basel, 4056, Switzerland
}

\begin{abstract}
The literature is constalleted with a wide variety of chemosensors against a plethora of analytes. $[1,2,3,4,5,6,7,8,9,10]$ This seminal library is used to inspire chemists to improve them using chemical synthesis. However, their optimization via chemical synthesis is a difficult task which takes time without the guarantee of final success. [11] We show here that combinatorial chemistry [12], the use of first and second coordination spheres and the displacement of indicators united within a protein cavity [13] offers an easy-to-assemble colorimetric bio-chemical sensor. It consists only of commercial chemicals. This colorimetric sensor is highly modular, cheap and evolvable. Its Xray structure reveals the composition of its active site. This allows to design it rationally for the recognition of dopamine with the naked eye. Our bio-sensor therefore resembles a biological receptor for the recognition of neurotransmitters. Its immediate high adaptability and ability to be evolved can be useful for the selective detection of a wide variety of analytes going from small molecules to microorganisms. This discovery therefore makes it possible to dream of new biotechnological or new immunotherapeutic applications. $[14,15,16,17,18,19,20,21,22]$
\end{abstract}

\section{Introduction}

The literature abounds with descriptions of chemo-sensors for the detection of a wide variety of analytes. [1, $2,3,4,5,6,7,23]$ In particular be made of: cyclophanes [24], crown ethers [25, 26], cryptands [27], cavitands [28, 29], calix[n]arenes [30], calix[n]pyrroles [31], cucurbit[n]uril [32], cyclodextrines [33, 34], pinwheels [35,36], dendrimers [37] or MOFS [38]. All these sensors take advantage of the first coordination sphere to capture analytes. None of them are easily optimized. Each requires synthesis steps that take time, are tedious and do not guarantee success. Based on this, by observing Nature more closely, we realize that bio-receptors such as membrane receptors $[39,40]$ are perfectly designed for the recognition of analytes. [40] Mention may in particular be made of membrane receptors for the recognition of neurotransmitters, for example dopaminergic receptors. [40, 41]

These optimized receptors $[42,43]$ take advantage of the second coordination sphere given by a protein environment for selective recognition. The active recognition site is decorated with amino acids that interact with the analyte to hold it captive. Therefore, inspired by chemical and biological sensors, we wanted to merge the best of both worlds to make a novel type of sensor. A sensors optimized by the first

\footnotetext{
${ }^{1}$ Corresponding author.

2E-mail: thibaud.rossel@emsp.gfbienne.ch

${ }^{3}$ E-mail: bing.zhang@unibas.ch
} 
and second sphere of coordination for the exquisite recognition of analytes. In addition, we wanted to add optical properties to it, in particular a color change during detection by using an indicator displacement.

We chose a protein with a deep cavity. [13] We then redesigned its active site by introducing a ligand into it. This ligand carries a functional group and is anchored supramolecularly to the protein. On this functional group we added a colored indicator. When an analyte enters the active site, it attaches to the functional group and displaces the indicator. The change in color indicates interactions between the active site and the analyte. The second sphere of coordination acts as a screen. It selects the access to the analyte at the active site. More specifically, we have incorporated into the carbonic anhydrase, boronic acids $[2,3,5,7]$ coordinated with indicators. These colorimetric sensors were screened in the presence of neurotransmitters.

The results show that we have obtained a highly selective, cheap and easy to assemble dopamine sensor mimicking biological membrane receptors. Such a discovery allows us to imagine optimizations taking advantage of the broad possibilities such as the ligand and its fonctionnal group, the indicators, the protein by mutation, the $\mathrm{pH}$, the temperature and or the concentration of the reactions, etc. This can lead to dynamic combinatorial chemistry [12], high throughput screening [44] and optimization processes via directed evolution protocols [45] using for example machine learning [46]. The immediate high adaptability and ability of our sensor to be evolved can be useful for the selective detection of a wide variety of analytes going from small molecules to microorganisms. This discovery therefore makes it possible to dream of new biotechnological or new immunotherapeutic applications $[14,15,16,17,18,19,20,21,22]$ We show, proof of principle, that guided through the crystal structure of our receptor, we can create a cheap, easy to assemble, colorimetric biosensor for the selective naked-eye detection of dopamine which mimics a dopaminergic receptor.

\section{Methods}

\subsection{First coordination sphere detection}

Dissolving 4-sulfamoyl(phenyl)boronic acid (1 p-BA, $250 \mu \mathrm{M})$ and pyrocatechol violet (2 PCV, $250 \mu \mathrm{M})$ in 2-[4-(2- hydroxyethyl)-1 piperazinyl]ethanesulfonicacid (HEPES, 100mM, pH=7.4) in a 1:1 mixture formed ( $p$-BAPCV, $250 \mu \mathrm{M}$ ), which is water-soluble and orange-red at working micromolar concentrations (see Figure 1). By homology with other reported arylboronic acid described in literature, we hypothesized that $p$-BAPCV could be formed and used to assemble a receptor for the naked-eye detection of various vicinal diols such as carbohydrates as described for 17 (D)-fructose [2] (See Figure 3) or catechols [1] such as 9 dopamine (see Figure 1 and Figure 3).

As anticipated $p$-BAPCV can detect visually and spectrophotometrically both 17 (D)-Fructose and 9 dopamine with a concomitant color change using an indicator displacement assay (IDA) (see Figure 1). We determined the association constant of $p$-BAPCV with 9 dopamine using an indicator displacement assay and we obtained a $\mathrm{K}_{\text {ass }}=2.12 \cdot 10^{6} \mathrm{M}^{-1}$ (see Table 1). [47] [2] Screening of various $\mathrm{pHs}$ ranging from $\mathrm{pH}=5$ to $\mathrm{pH}=8$ showed the best discrimination at $\mathrm{pH}=7.4$ as reported in other publications. $[2,6]$

\subsubsection{Chemical diversity}

To generate diversity in the composition of our sensors, we tested and used chemical optimization strategies. In such, combining two different sulfonamides boronic acids (1 $p$-BA or $\mathbf{2} m$-BA) with catechol based indicators (3 PCV or 4 ARS) yielded four potential sensors for the detection of analytes. Of the four sensors tested only $\mathbf{1} p$-BA was able to coordinate either $\mathbf{3}$ PCV or $\mathbf{4}$ ARS forming: $p$-BAPCV and $p$-BAARS. $2 \mathrm{~m}$-BA did not reacted with both indicators suggesting that the position of the boronic acid in the aryl-sulphonamid moiety plays a key role in the coordination process of the indicator. With both $p$-BAPCV and $p$-BAARS in hands, we tested the two chemical variants with 17 (D)-Fructose for recognition and both offered an indicator displacement assay allowing detection of the analyte and showing no special selectivity in the recognition process (see Figure 3). For the continuation of our study, we thefore selected $p$-BAPCV and $p$-BAARS. 
A)

a)

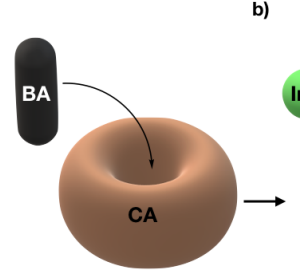

d)

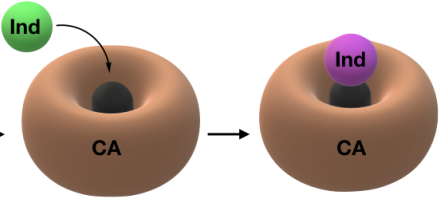

e)
B)

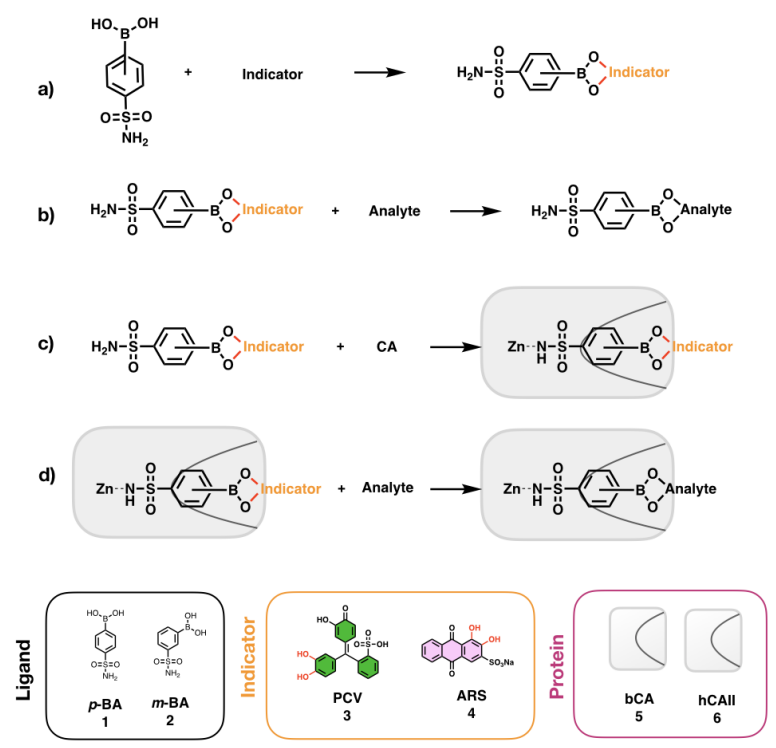

C)

a)

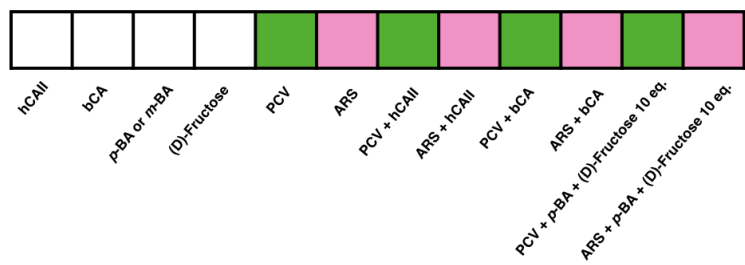

D) a)

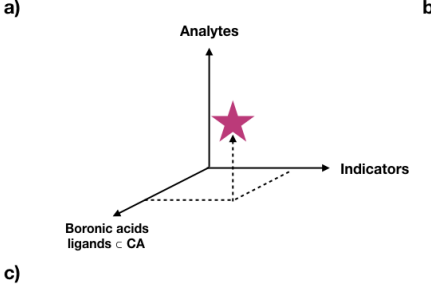

b)

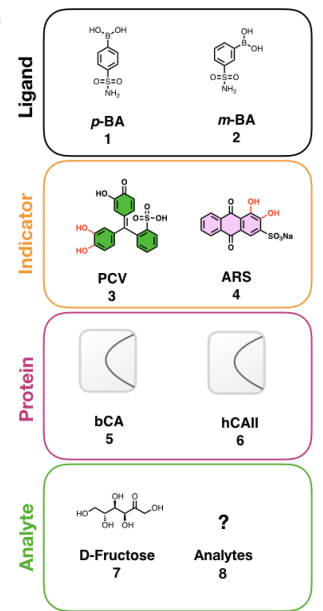

Figure 1. A) General principle of the supramolecular sensor: Addition of an arylsulfonamid boronic acid (BA) to carbonic anhydrase variants (CA) offers BA $\subset$ CA. Addtion of an indicator (Ind) to the supramolecular construct allow the creation of BAInd $\subset$ CA. Final addition of an analyte (An) allows an indicator displacement assay in the deep cavity of the protein liberating the indicator and forming BAInd C CA with a concomittant change in color B) Synthesis of the synthetic receptor; arylsulfonamidboronic acid $\mathbf{1}(250 \mu \mathrm{M})$ is added to catechol based indicator $\mathbf{3}$ or $4(250 \mu \mathrm{M})$ in a 1:1 mixture, a naked eye change in color is observed. Upon addition of CA (either 5 bCA and $6 \mathrm{hCAll}, 250 \mu \mathrm{M})$ to the mix, the synthetic receptor BAIndc CA (250 $\mu \mathrm{M})$ is formed and a change in color of the solution appears. The addition of analytes of interest 8 (10 eq., $2500 \mu \mathrm{M}$ ) allows a concomitant color change with an indicator displacement assay and forms BAAn $\subset$ CA and releases the indicator $\mathbf{3}$ or $\mathbf{4}$. C) a) When $\mathbf{1}$ or $\mathbf{2}$ is added to $\mathbf{3}$ or $\mathbf{4}$, a coordination may occur of the indicator to the boronic acid generating a change in color. Addition of $\mathbf{5}$ bCA to the construct forms a colored solution indicating the formation of BAlnd $\subset$ bCA. Addition of a 10 eq. of 9 dopamine allows an indicator displacement assay releasing the indicator and with a concomitant change in color b) Control experiments. D) Optimisation strategy for the creation of a library of biosensors b) Building blocks for the creation of a biosensor library c) Results of the optimization screening: $\mathbf{2}$ PCV is green in solution at $\mathrm{pH}=7.4(250 \mu \mathrm{M})$, upon addition of $1 p$-BA $250 \mu \mathrm{M}$, the solution changes colour to orange forming p-BAPCV. The addition to the mix of 6 human carbonic anhydrase II (250 $\mu \mathrm{M})$, generates a bathochromic shift in colour from orange to pink suggesting the entry of the complex inside the pocket of the protein and forming $p$-BAPCV $\subset$ hCAll. The addition of an excess of 17 (D)-fructose $(0.025 \mathrm{M}$, line $1 \mathrm{Cplx} 1 \mathrm{hCAll})$ to $p$-BAPCV $\subset$ hCAll doesn't allow a displacement of the indicator (3 PCV or 4 ARS) whereas outside of the protein (Line 1 Cplx : 0.1 hCAII) the indicator displacement assay occurs. It suggests that the protein prevents the entry of 17 (D)-fructose due to second coordination sphere interactions. 
Table 1. Association constants of systems

\begin{tabular}{ll}
\hline System & $\mathrm{K}_{\text {ass }}\left[\mathrm{M}^{-1}\right]$ \\
\hline$p$-BA with PCV & $1.36 \cdot 10^{5}$ \\
$p$-BAPCV with dopamine & $2.12 \cdot 10^{6}$ \\
$p$-BAPCV with bCA & $3.60 \cdot 10^{5}$ \\
$p$-BAPCV $\subset$ bCA with dopamine & $\mathrm{xxxxxxx}$ \\
\hline
\end{tabular}

\subsection{Second coordination sphere detection}

The catalytic Zn-active site present at the bottom of a deep hydrophobic funnel-shaped cavity of carbonic anhydrase endows high affinity for para-substituted aryl sulphonamids. [13] We thus speculated that upon tethering an aryl-sulfonamides boronic acid conjugated with an indicator may allow to anchor a recognition site within $\mathbf{5} \mathrm{bCA}$ allowing an exchange between the indicator and selected molecules of choice (see Figure 1). In a first time, in order to confirm our hypothesis, we assembled the arylboronic acid $1 p$-BA $(250 \mu \mathrm{M})$ and 2 PCV $(250 \mu \mathrm{M})$ in HEPES $100 \mathrm{mM}, \mathrm{pH}=7.4$ and formed $p$-BAPCV $\left(\lambda_{\max }=465 \mathrm{~nm}\right)$ which is orange colour in a 1:1 mixture. We performed a titration of $\mathbf{1}$ with $\mathbf{2}$ in order to determine the binding constant of the host-guest receptor and obtained a $\mathrm{K}_{\text {ass }}=1.36 \cdot 10^{5} \mathrm{M}^{-1}$ (see Table 1) with a 1:1 association.

In a second time, we added 5 bCA ( 1 eq.) to the $1: 1$ mix of $p$-BAPCV or $p$-BAARS ( $250 \mu \mathrm{M}$ in HEPES $\mathrm{pH}=$ 7.4). For $p$-BAPCV $\subset$ bCA, a bathochromic change in colour appeared immediately after the addition of the protein (see Figure 1). The orange solution $\left(\lambda_{\max }\right.$ of $=495 \mathrm{~nm}$ ) of $p$-BAPCV turned intense pink with a $\lambda_{\max }$ of $=525 \mathrm{~nm}$ (see Figures 1 and 3). Titration of $5 \mathrm{bCA}$ with $p$-BAPCV shows a batochromic shift in color from $495 \mathrm{~nm}$ to $525 \mathrm{~nm}$ and a Job Plot titration confirms a 1:1 association of the bio-receptor (see supporting information) and an association constant of $3.60 \cdot 10^{5} \mathrm{M}^{-1}$ (seeTable 1). Same conclusions where observed for $p$-BAARS $\subset$ where the shift in color is from $480 \mathrm{~nm}$ for $p$-BAARS to $485 \mathrm{~nm}$ for $p$-BAARS $c$ however less pronounced than for $p-B A P C V \subset b C A$. The change in color confirms that the assembled orange colored p-BAPCV enters the protein. More interestingly, the order of the addition of $1 p$-BA, $\mathbf{3}$ PCV and the protein 5 bCA didn't play any critical role. One can either form $p$-BAPCV before addition to $\mathbf{5}$ bCA or add $p$-BAPCV to the protein and add the indicator such as $\mathbf{3}$ PCV in a second time for coordination to $p$-BAPCV (see Figures 1 and 3) leading to an indicator uptake-assay which is known to improve the sensitivity of the assay compared to the displacement. [23] It suggests that CA can be used as a boronic proteic based platform for the recognition of analytes opening new routes for therapeutic proteins against catecholamines and that $\mathbf{3}$ PCV can be used as a sensor to probe the recognition of analytes coordinated to the boronic acid inside the active site of the protein.

\subsubsection{Proteic diversity}

In order to increase the diversity and induce selectivity in our sensors, we combined the two constructs $p$-BAPCV and $p$-BAARS with two variants of carbonic anhydrase such as $\mathbf{5}$ bCA (bovine carbonic anhydrase) and $\mathbf{6}$ hCAll (human carbonic anhydrase). The combination offered a four possibilities matrix ready to be screened with analytes; $p$-BAPCV $\subset$ bCA, $p$-BAPCV $\subset$ hCAll, $p$-BAARS $\subset$ bCA, $p$-BAARS $\subset$ hCAIl.

\subsection{Indicator displacement assay}

We then tested our matrix of four sensors; $p$-BAPCV $\subset$ bCA, $p$-BAPCV $\subset$ hCAll, $p$-BAARS $\subset$ bCA, $p$-BAARS $\subset$ hCAll for the detection of $\mathbf{1 7}$ (D)-fructose troughs an IDA since recognition is described in literature outside a second coordination sphere. [2] The detection was not possible even in the presence of a large excess of 17 (D)-fructose (100 eq. of fructose compared to the proteic bio-congugates) (see Figure 3 ) thus suggesting that the cavity of the protein prevents the entry of the sugar compared to the free assembled ligand-boronic complex $p$-BAPCV. In order to confirm the supra-molecular assembly, we performed a titration of the complex $p$-BAARS and $p$-BAPCV with a $1(250 \mu \mathrm{M})$ to $0.1(25 \mu \mathrm{M})$ ratio of $p$-BAARS and $p$-BAPCV versus 5 bCA and an excess of 17 (D)-fructose (100 eq., $0.025 \mathrm{M}$ ) (see Figure 1). Colour change 
outside the protein was observed both for $\mathbf{3}$ ARS and $\mathbf{4}$ PCV as indicators confirming that the excess of $p$-BAARS and $p$-BAPCV $(250 \mu \mathrm{M})$ outside the protein reacts with 17 (D)-fructose (seefigures 1 and 3). As a consequence and in order to explore further the detection ability of our construct, we decided to investigate various analytes for recognition.

\subsection{Crystal structure of the bio-sensor}

In order to do so and predict further the interactions of potential analytes with our biosensor, we set out to crystallize it. We builded crystals of hCAll according to published procedures [48] and soaked 1 $p$-BA inside the enzyme. We obtained a crystal structure of our sensor $p$-BA $\subset$ hCAll (PDB $=6 \mathrm{XVH}$, see Figure $2 \mathbf{A}$ and $\mathbf{B}$ ) with same procedure also reported other crystal structures [48] of hCAll in conjugation with inhibitors [13] and in particular the crystal structure of hCAll with 18 4-(4,4,5,5-tetramethyl-1,3,2dioxaborolan-2-yl)phenylsulfamide $[16,17,39,49,50,40,51,48]$ (PDB $=3 \mathrm{MNU}$, see Figure 2). Attempt to obtain crystal structure of $p$-BA $\subset$ hCAll in conjugation with 3 PCV or 9 dopamine was not possible probably due to disruption of crystals in the soaking process.

\subsection{Screening of analytes}

With the obtained crystal structure (PDB $=6 \mathrm{XVH}$ ) in hands (see Figure 2 ) we reasoned that molecules with a geometric similarity with the cavity should enter more easily the pocket of CA compared to 17 (D)-fructose. [2] In so, we were particularly interested in the close lying aminoacids to the boronic acids moiety such as: T-200, L-198, F-131, V-135, C-206. F-131 and especially F-131 that could interact with potential tested analytes entering the activ site of our construct.

In addition, for screening of analytes of interest, we concentrated our efforts towards medically relevant and compatibles geometries molecules such as : 9 dopamine, $10(S)$-DOPA, $11(R)$-norepinephrine, 12 (R)-epinephrine, 13 GABA, 14 (S)-glutamic acid, 15 homovanilic acid, 16 DOPAC, 17 (D)-Fructose. (see Figure 3). Finally, we selected $p$-BAPCV $\subset$ bCA and $p$-BAPCV $\subset$ hCA II for screening since best discrimination with the naked eye is obtained with these systems (going from pink to green).

\section{Results}

The results of the screening showed that $p$-BAPCV $(250 \mu \mathrm{M})$ is able to recognize the catechol based neurotransmitters tested (small excess of 10 eq., $2500 \mu \mathrm{M}$ ) showing no special selectivity (see Figure 1 and Figure 3). 13 GABA or 14 glutamic acid for example where not recognized even with a large excess of analyte (10 or 100 eq.). $p$-BAPCV $\subset$ bCA and $p$-BAPCV $\subset$ hCAll are not able to recognize 17 (D)-fructose even with a large excess (100 eq. compared to the bioreceptor $p$-BAPCV) suggesting that the second coordination sphere plays a critical role in preventing the recognition of the analyte (seefigures 1 and 3 ). $p$-BAPCV $\subset$ bCA and $p$-BAPCV $\subset$ hCAll recognize preferentially $\mathbf{9}$ dopamine over $\mathbf{1 1}(R)$-norephinephrine or $\mathbf{1 2}$ $(R)$-ephinephrine and other catechol based neurotransmitter even with significant structural similarities suggesting that second coordination sphere plays an Determining role in the selectivity process in the cavity of the protein (see Figure 3). Hence, 15 Homovanilic acid is not recognized by either $p$-BAPCV $\subset$ bCA or $p$-BAPCV $\subset$ hCAll suggesting that the catechol moiety plays a critical role for the recognition of the analyte. $p$-BAPCV $\subset$ bCA $I$ and $p$-BAPCV $\subset$ hCAll follows the same trend in recognition of analytes suggesting that the active sites of both proteins are similar and pursue similar recognition patterns (see Figure 3). 9 dopamine is preferentially recognized over 16 DOPAC suggesting that the functional group at the tale of the neurotransmitter interacts with the second coordination of CA. A positive charge plays a key role in the recognition process (9 dopamine) over a negative charge (16 DOPAC).

\subsection{Detection of analytes discussion}

In order to understand the mechanism of selection discriminating the recognition between 9 dopamine and 16 DOPAC we sought to manually dock 9 dopamine inside $p$-BA $\subset$ hCAll. Upon manual bond rotation we found that F-131 was close lying to the tail of 9 dopamine (see Figure 2). We then realized that the close proximity of an aromatic ring to an amine may lead to an $\mathrm{NH} / \pi$ interaction that could stabilize the 
A)

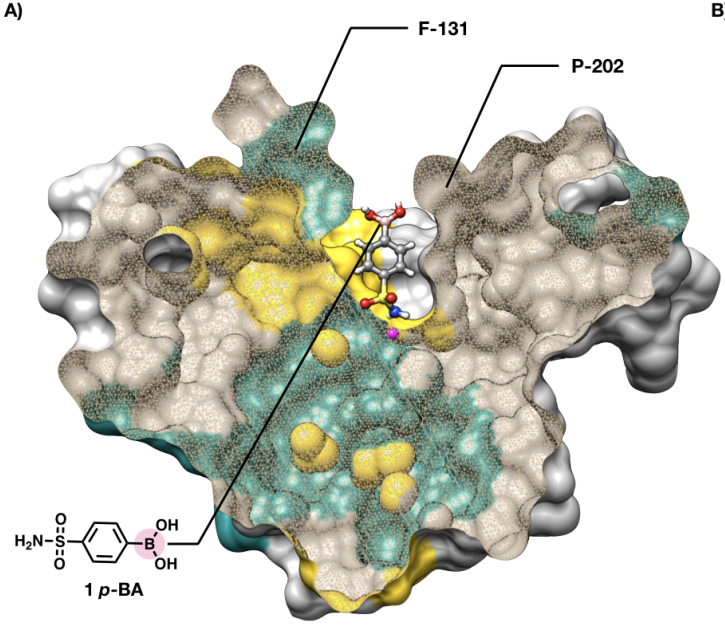

c)

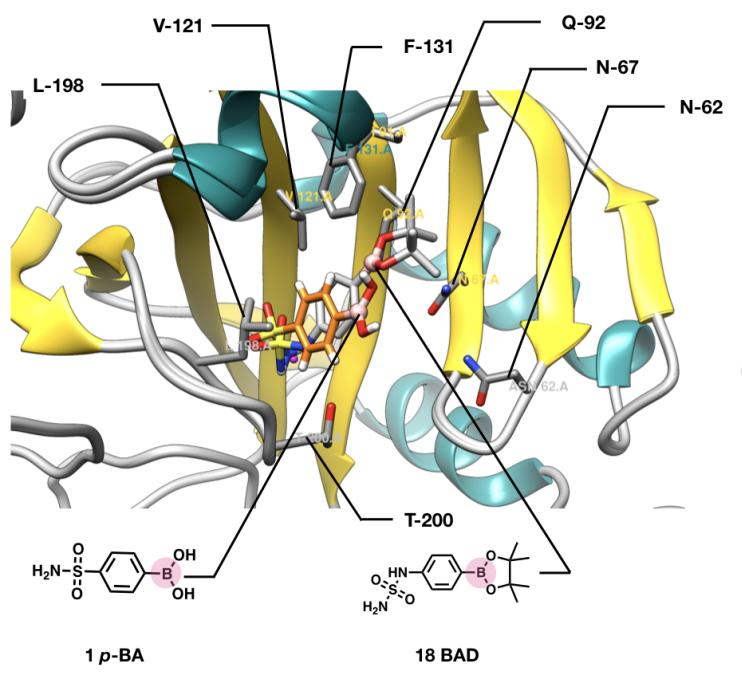

D)

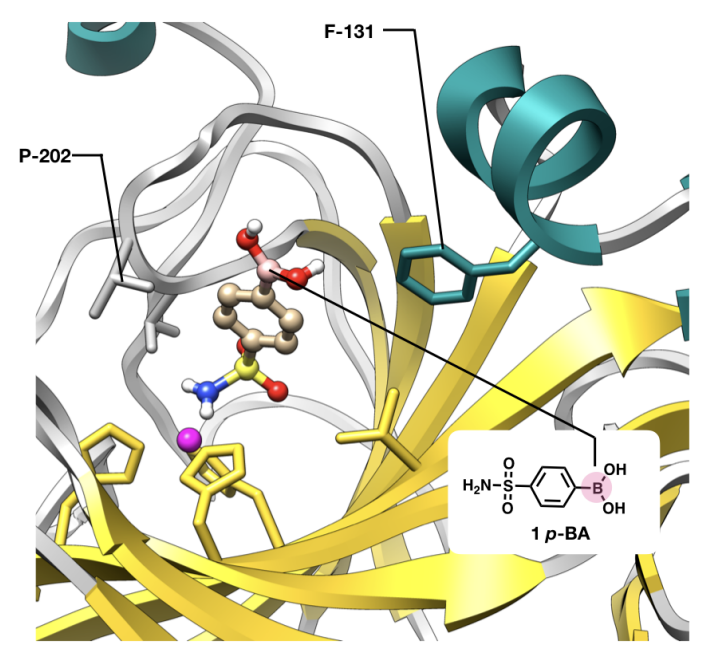

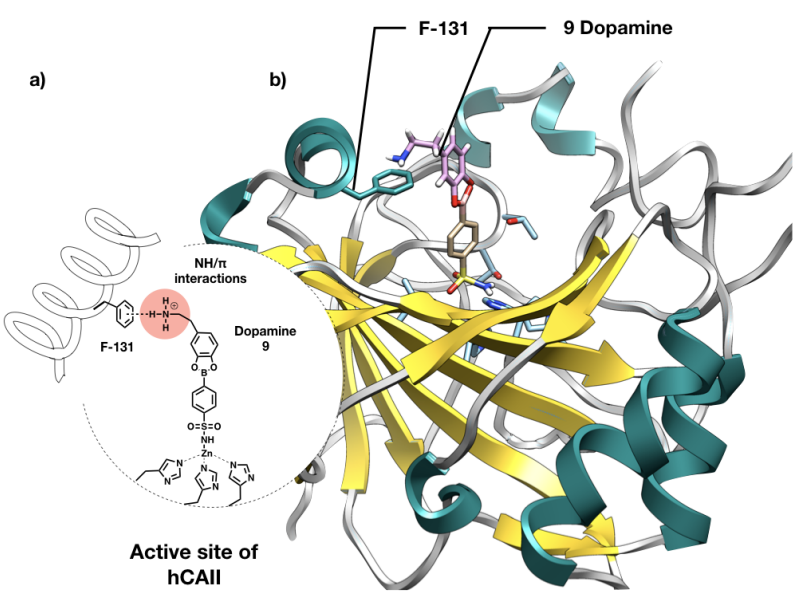

Figure 2. A) Cutted X-ray structure (PDB: $6 \mathrm{XVH}$ ) presenting $1 p$-BA inside hCAll. [48] Of particular interest is the close lying aminoacid (F-131) to the boronic acid center of the supramolecular construct. B) Close view of $p$-BA inside hCAll C) Superposition of hCAll in conjugation with 18 BAD (PDB code = 3MNU) and hCAll with $1 p$-BA (PDB code $=6 \mathrm{XVH}$ ). Of particular interest are the aminoacids close to the binding site such as N-62, T-200, V-121, F-131. D) a) Proposed mechanisms for the recognition of dopamine inside $p$-BA $\subset$ hCAll, a cataionic $\mathrm{NH} / \pi$ should occurs with close proximity of F-131 b) Manual docking of dopamine inside the active site of $p$-BA $\subset$ hCAll.

interaction between 9 dopamine and $p$-BA $\subset$ hCAll. We therefore suggest a mechanism of recognition with an $\mathrm{NH} / \pi$ interaction F-131 (See Figure 3).

\subsection{Test of the best sensor in a complex sample}

In order to test our best sensor $p$-BAPCV $\subset$ bCA in real conditions, we decided test the determination of 9 dopamine in human urine. Urine is known to contain traces of $\mathbf{9}$ dopamine due to metabolic activities. In a first time, we tested $p$-BAPCV in the presence of pure urine from two different persons. Naked-eye detection of 9 dopamine was not possible nor any disturbance appeared on the sensor. In a second time we built $p$-BAPCV $\subset$ bCA and tested it against the detection of traces of 9 dopamine in urine. No change in color was observable. In a third time, we spiked urine with $\mathbf{9}$ dopamine at a concentration of $2500 \mu \mathrm{M}$ 
A)

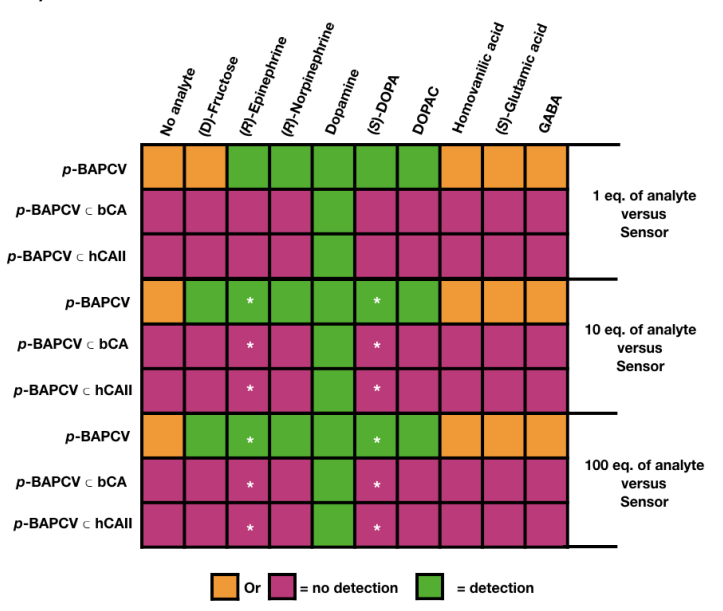

c)

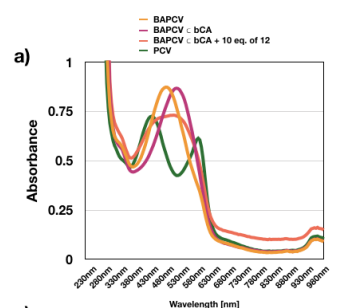

c)

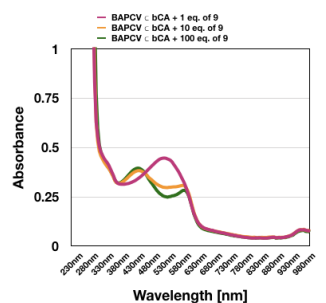

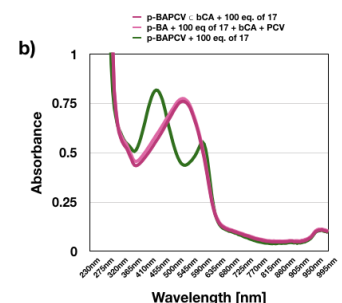

d)

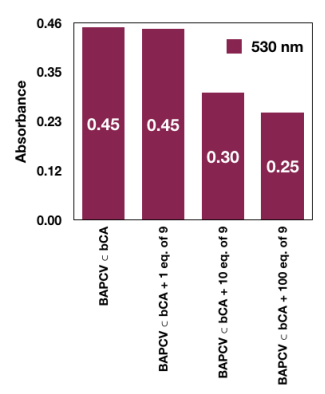

B)

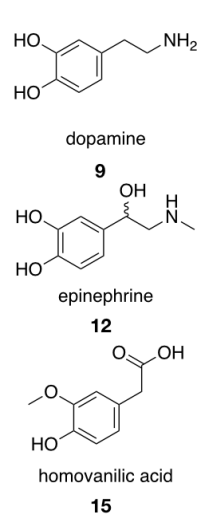

15

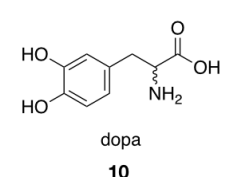

10<smiles>CC(C)(O)OC(=O)O</smiles>

16

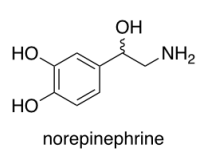

11<smiles>N[C@@H](CCC(=O)O)C(=O)O</smiles>

14

算

fructose
D) b)
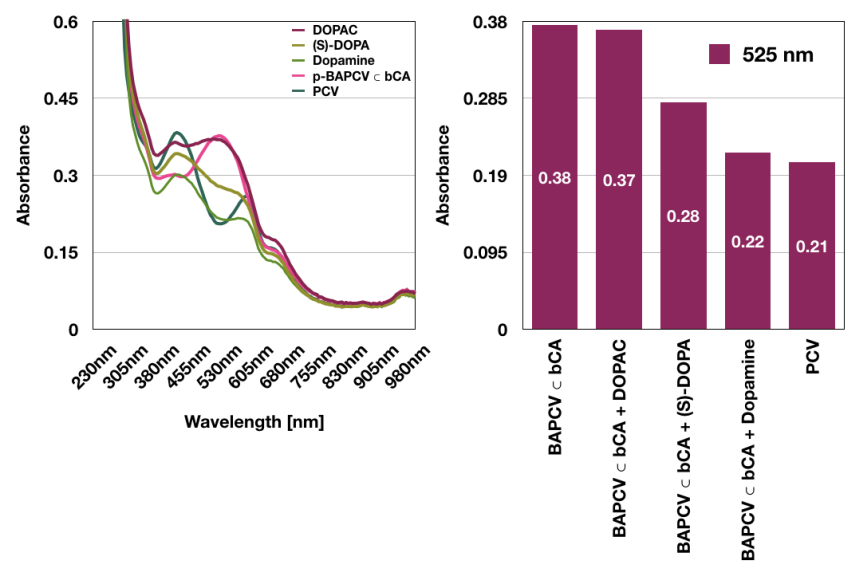

Figure 3. A) Screening of the synthetic bio-inspired receptor $p$-BAPCV $\subset$ bCA for recognition of neurotransmitters. $p$-BAPCV $(250 \mu \mathrm{M})$, outside the protein is orange whereas inside the protein (CA, $250 \mu \mathrm{M})$, the construct ( $p$-BAPCV $\subset$ bCA) is pink. Addition of a small excess ( $2500 \mu \mathrm{M}, 10$ eq. versus sensor) of the analyte to the boronic acid outside of the protein may allow an indicator displacement assay. When $p$-BAPCV $(250 \mu \mathrm{M})$ is incorporated inside either 5 bCA or 6 hCA II $(250 \mu \mathrm{M})$ forming $p$-BAPCV $\subset$ bCA only 9 dopamine can be fully recognized even with a large excess ( $0.025 \mathrm{M}, 100$ eq.) of the other analytes. B) Analytes used in the screening of this paper C) Absorbance spectras of the screening a) the incorporation of $p$-BAPCV $(250 \mu \mathrm{M})$ into bCA $(250 \mu \mathrm{M})$ allows a bathochromic shift in colour from $\lambda_{\max }=495 \mathrm{~nm}$ to $\lambda_{\max }=525 \mathrm{~nm}$. Recognition of $(R)$-epinephrine 11 (100 eq., $0.025 \mathrm{M}$ ) at a concentration 100 eq. higher than $\mathbf{5} p$-BA allows a poor recognition $\mathrm{b}$ ) addition of $\mathbf{1 7}$ (D)-fructose to $p$-BAPCV $\subset$ bCA doesn't allows an IDA either c) addition of a small excess (10 eq., $2500 \mu \mathrm{M}$ ) of dopamine 9 to $p$-BAPCV $\subset$ bCA allows an indicator displacement assay d) Decreasing absorbance upon addition of various catechol based analytes such of 9 dopamine (from 1 eq. to 100 eq.) to $p$-BAPCV $\subset$ bCA D) a) Absorbance spectras of $p$-BAPCV $\subset$ bCA in addition with various analytes b) Absorbance of $p$-BAPCV $\subset$ bCA with various analytes at $525 \mathrm{~nm}$. 
and tested $p$-BAPCV $\subset$ bCA for recognition. Change in color appeared immediately proving the possible determination of $\mathbf{9}$ dopamine inside complex samples such as human urine. (See supporting information)

\section{Conclusions}

Bio-molecular systems such as enzymes normally are dedicated to substrate transformations and allows very specific catalytic processes that depends on the composition of the active site. Here we have shown that such an active site can be completely redesigned by the introduction of a specific ligand bearing a functional group. In specific, we have shown that boronic acids can be introduced supramolecularly into a proteic environment and coordinated to indicators allowing specific detection of analytes using a displacement assay, for example dopamine. Despite their limited detection limits, these bio-receptors are distinguished by their easy to assemble, commercial disponibility, high modularity and evolvable features. Key to our construction strategy was the reimagination of the active site of carbonic anhydrase within a new structural context; a new boronic acid ligand for coordination of various analytes surrounded by a selectiv second coordination sphere. This example expands the genernal use of enzymes transforming them into exquisite biosensors. It could potentially be used for detection of various analytes going from small molecules to microorganisms mimicking natural antibodies.

\section{Competing interests}

The authors declare no competing interests

\section{Contributions}

Thibaud Rossel conducted the research, performed the experiments and wrote the manuscript. Bing Zhang produced crystals solved the X-ray structure. Raphael Gobat helped with the crystals production and with solving the X-ray structure.

\section{Experimental section}

All chemicals were puchased by Sigma Aldrich and used without further purifications. hCAll was synthesized by the Ward group University of Basel Switzerland.

A typical experiment (such as Figure $\mathbf{3 C}$ and $\mathbf{D}$ is realised as follow (all solutions are freshly prepared prior to experiment):

$25 \mu \mathrm{L}$ of a $250 \mu \mathrm{M}$ solution of BA in HEPES pH $=7.4100 \mathrm{mM}$ is added to a $25 \mu \mathrm{L}$ of a $250 \mu \mathrm{M}$ solution of indicator in a 96 well plate (same buffer). Uppon change in color, $25 \mu \mathrm{L}$ of CA is added to the mix and change in color is observed (same buffer). Addition of $25 \mu \mathrm{L}$ of analyte allows an indicator displacement assay and a change in color. The absorbance spectra of the wells are measured in a 96 well plate reader TECAN safire 2 for analysis and comparison.

\section{Acknowledgements}

We thanks Prof. Camilo Perez as well as Prof. Gilles Gasser for fruitfull discussions in this project. We thank Prof. Thomas R. Ward for a loan of a spectrophotometer as well as hCAll. We thank the analytical service (NPAC) of the University of Neuchâtel as well as the Gymnase français de Bienne for providing us lab equipment.

\section{References}

[1] L. Zhu, Z. Zhong, and E. V. Anslyn. Guidelines in implementing enantioselective indicator-displacement assays for $\alpha$-hydroxycarboxylates and diols. Journal of the American Chemical Society, 127(12):42604269, 2005. 
[2] G. Springsteen and B. Wang. Alizarin Red S as a general optical reporter for studying the binding of boronic acids with carbohydrates. Chemical Communications, 17:1608-1609, 2001.

[3] Z. Wu, M. Li, H. Fang, and B. Wang. A new boronic acid based fluorescent reporter for catechol. Bioorganic \& medicinal chemistry letters, 22(23):7179-7182, 2012.

[4] K. E. Secor and T. E. Glass. Selective amine recognition: development of a chemosensor for dopamine and norepinephrine. Organic letters, 6(21):3727-3730, 2004.

[5] X. T. Zhang, G. J. Liu, Z. W. Ning, and G. W. Xing. Boronic acid-based chemical sensors for saccharides. Carbohydrate research, 452:129-148, 2017.

[6] L. Zhu, Z. Zhong, and E. V. Anslyn. Guidelines in implementing enantioselective indicator-displacement assays for $\alpha$-hydroxycarboxylates and diols. Journal of the American Chemical Society, 127(12):42604269, 2005.

[7] Z. Guo, I. Shin, and J. Yoon. Recognition and sensing of various species using boronic acid derivatives. Chemical Communications, 48(48):5956-5967, 2012.

[8] T. Rossel and M. Creus. A biomimetic cerium-based biosensor for the direct visual detection of phosphate under physiological conditions. Chemical Communications, 55(99):14894-14897, 2019.

[9] T. Rossel and M. Creus. «La Chimie en Couleurs»: Socially Relevant \& Original Research in Chemistry in High Schools Using Modest Resources. CHIMIA International Journal for Chemistry, 73(7-8):599-603, 2019.

[10] X. Wu, X. X. Chen, and Y. B. Jiang. Recent advances in boronic acid-based optical chemosensors. Analyst, 142(9):1403-1414, 2017.

[11] R. A. Tromans, T. S. Carter, L. Chabanne, M. P. Crump, H. Li, J. V. Matlock, M. G. Orchard, and A. P. Davis. A biomimetic receptor for glucose. Nature chemistry, 11(1):52, 2019.

[12] J. M. Lehn and A. V. Eliseev. Dynamic combinatorial chemistry. Science, 291(5512):2331-2332, 2001.

[13] V. M. Krishnamurthy, G. K. Kaufman, A. R. Urbach, I. Gitlin, K. L. Gudiksen, D. B. Weibel, and G. M. Whitesides. Carbonic anhydrase as a model for biophysical and physical-organic studies of proteins and protein- ligand binding. Chemical reviews, 108(3):946-1051, 2008.

[14] A. Barbieri, S. Bimonte, G. Palma, A. Luciano, D. Rea, A. Giudice, and O. De Cobelli. The stress hormone norepinephrine increases migration of prostate cancer cells. International journal of oncology, 47:527534, 2015.

[15] K. Guo, Q. Ma, L. Wang, H. Hu, J. Li, D. Zhang, and M. Zhang. Norepinephrine-induced invasion by pancreatic cancer cells is inhibited by propranolol. Oncology reports, 22(4):825-830, 2009.

[16] D. Palm, K. Lang, B. Niggemann, T. L Drell IV, K. Masur, K. S. Zaenker, and F. Entschladen. The norepinephrine-driven metastasis development of PC-3 human prostate cancer cells in BALB/C nude mice is inhibited by $\beta$-blockers. International journal of cancer, 118(11):2744-2749, 2006.

[17] K. Lang, T. L. Drell IV, A. Lindecke, B. Niggemann, C. Kaltschmidt, K. S. Zaenker, and F. Entschlafen. Induction of a metastatogenic tumor cell type by neurotransmitters and its pharmacological inhibition by established drugs. International journal of cancer, 112(2):231-238, 2004.

[18] E. K. Sloan, S. J. Priceman, B. F. Cox, S. Yu, M. A. Pimentel, V. Tangkanangnukul, and A. K. Sood. The sympathetic nervous system induces a metastatic switch in primary breast cancer. Cancer research, 70(18):7042-7052, 2010. 
[19] F. Entschladen, I. V. Drell, L. Theodore, K. Lang, J. Joseph, and K. S. Zaenker. Neurotransmitters and chemokines regulate tumor cell migration: potential for a new pharmacological approach to inhibit invasion and metastasis development. Current pharmaceutical, 11(3):403-411, 2005.

[20] A. C. Walls, M. A. Tortorici, B. J. Bosch, B. Frenz, P. JM Rottier, F. DiMaio, F. A. Rey, and D. Veesler. Cryoelectron microscopy structure of a coronavirus spike glycoprotein trimer. Nature, 531(7592):114-117, 2016.

[21] C. C. Lai, T. P. Shih, W. C. Ko, H. J. Tang, and P. R. Hsueh. Severe acute respiratory syndrome coronavirus 2 (SARS-CoV-2) and corona virus disease-2019 (COVID-19): the epidemic and the challenges. International journal of antimicrobial agents, page 105924, 2020.

[22] L. van der Hoek, K. Pyrc, M. F. Jebbink, W. Vermeulen-Oost, R. JM. Berkhout, K. C. Wolthers, P. ME. Wertheim van Dillen, J. Kaandorp, J. Spaargaren, and B. Berkhout. Identification of a new human coronavirus. Nature medicine, 10(4):368-373, 2004.

[23] B. E. Collins and E. V. Anslyn. Pattern-Based Peptide Recognition. Chemistry-A European Journal, 13(17):4700-4708, 2007.

[24] Danaboyina Ramaiah, Prakash P Neelakandan, Akhil K Nair, and Rekha R Avirah. Functional cyclophanes: promising hosts for optical biomolecular recognition. Chemical Society Reviews, 39(11):41584168, 2010.

[25] C. J. Pedersen. Cyclic polyethers and their complexes with metal salts. Journal of the American Chemical Society, 89(26):7017-7036, 1967.

[26] G. W. Gokel, W. M. Leevy, and M. E. Weber. Crown ethers: sensors for ions and molecular scaffolds for materials and biological models. Chemical reviews, 104(5):2723-2750, 2004.

[27] S. O. Kang, J. M. Llinares, V. W. Day, and Kristin Bowman-James. Cryptand-like anion receptors. Chemical Society Reviews, 39(10):3980-4003, 2010.

[28] D. J. Cram. Cavitands: organic hosts with enforced cavities. Science, pages 1177-1183, 1983.

[29] J. R. Moran, S Karbach, and D. J. Cram. Cavitands: synthetic molecular vessels. Journal of the American Chemical Society, 104(21):5826-5828, 1982.

[30] C. D. Gutsche and R. Muthukrishnan. Calixarenes. 1. Analysis of the product mixtures produced by the base-catalyzed condensation of formaldehyde with para-substituted phenols. The Journal of Organic Chemistry, 43(25):4905-4906, 1978.

[31] P. .A Gale, J. L. Sessler, V. Kral, and V. Lynch. Calix [4] pyrroles: Old yet new anion-binding agents. Journal of the American Chemical Society, 118(21):5140-5141, 1996.

[32] W. A. Freeman, W. L. Mock, and N. Y. Shih. Cucurbituril. Journal of the American Chemical Society, 103(24):7367-7368, 1981.

[33] A. Villiers. Bull. Soc. Chim. Paris, 45:468, 1891.

[34] Gregorio Crini. A history of cyclodextrins. Chemical reviews, 114(21):10940-10975, 2014.

[35] A. Metzger, V. M. Lynch, and E. V. Anslyn. A synthetic receptor selective for citrate. Angewandte Chemie International Edition in English, 36(8):862-865, 1997.

[36] C. Schmuck and M. Schwegmann. A molecular flytrap for the selective binding of citrate and other tricarboxylates in water. Journal of the American Chemical Society, 127(10):3373-3379, 2005.

[37] E. Buhleier, W. Wehner, F. Vögtle, et al. "Cascade"- and "nonskid-chain-like" synthesis of molecular cavity topologies. Synthesis, 9(2):155-158, 1978. 
[38] H. Li, M. Eddaoudi, M. O'Keeffe, and O. M. Yaghi. Design and synthesis of an exceptionally stable and highly porous metal-organic framework. Nature, 402(6759):276-279, 1999.

[39] A. Nieoullon. Dopamine and the regulation of cognition and attention. Progress in neurobiology, 67:53-83, 2002.

[40] M. Y. S. Kalani, N. Vaidehi, S. E. Hall, R. J. Trabanino, P. L. Freddolino, M. A. Kalani, and W. A. Goddard. The predicted 3D structure of the human D2 dopamine receptor and the binding site and binding affinities for agonists and antagonists. Proceedings of the national academy of sciences, 101:3815-3820, 2004.

[41] R. A. Wise. Dopamine, learning and motivation. Nature reviews. neuroscience, 5(6):483, 2004.

[42] J. M. Beaulieu and R. R. Gainetdinov. The physiology, signaling, and pharmacology of dopamine receptors. Pharmacological reviews, 63(1):182-217, 2011.

[43] R. A. Nicoll, R. C. Malenka, and J. A. Kauer. Functional comparison of neurotransmitter receptor subtypes in mammalian central nervous system. Physiological reviews, 70(2):513-565, 1990.

[44] J. Bajorath. Integration of virtual and high-throughput screening. Nature Reviews Drug Discovery, 1(11):882, 2002.

[45] F. H. Arnold. Design by directed evolution. Accounts of chemical research, 31(3):125-131, 1998.

[46] K. K. Yang, Z. Wu, and F. H. Arnold. Machine-learning-guided directed evolution for protein engineering. Nature methods, 16(8):687-694, 2019.

[47] Pall Thordarson. Determining association constants from titration experiments in supramolecular chemistry. Chemical Society Reviews, 40(3):1305-1323, 2011.

[48] G. De Simone and C. T. Supuran. Carbonic anhydrase IX: Biochemical and crystallographic characterization of a novel antitumor target. Biochimica et Biophysica Acta (BBA)-Proteins and. Proteomics, 1804(2):404-409, 2010.

[49] D. D. Dougherty, A. A. Bonab, T. J. Spencer, S. L. Rauch, B. K. Madras, and A. J. Fischman. Dopamine transporter density in patients with attention deficit hyperactivity disorder. The Lancet, 354(9196):21322133, 1999.

[50] K. Lang, T.L. DRELL IV, A. Lindecke, B. Niggemann, C. Kaltschmidt, K. S. Zaenker, and F. Entschladen. Induction of a metastatogenic tumor cell type by neurotransmitters and its pharmacological inhibition by established drugs. International journal of cancer, 112(2):231-238, 2004.

[51] G. Li, Y. Dong, and M. T. Reetz. Can machine learning revolutionize directed evolution of selective enzymes? Advanced Synthesis \& Catalysis, 361(11):2377-2386, 2019. 